\title{
Desempenho produtivo e eficiência bioeconômica de bovinos Nelore e Caracu selecionados para peso aos 378 dias de idade recebendo alimentação à vontade ou restrita ${ }^{1}$
}

\section{Antonio Gesualdi Júnior ${ }^{2}$, Augusto César de Queiroz ${ }^{3}$, Flávio Dutra de Resende ${ }^{4}$, Guilherme Fernando Alleoni ${ }^{5}$, Alexander George Razook ${ }^{6}$, Leopoldo Andrade de Figueiredo ${ }^{6}$, Ana Cristina Ladeira de Souza Gesualdi ${ }^{7}$, Edenio Detmann ${ }^{3}$}

\footnotetext{
${ }_{1}^{1}$ Parte da tese de Doutorado do primeiro autor, realizada no APTA - SP e UFV - MG. Projeto financiado pela FAPESP.

2 Parque de Alta Tecnologia do Norte Fluminense - TECNORTE, Av. Alberto Lamego 2000, Campos dos Goytacazes - RJ, CEP: $28013-602$.

${ }^{3}$ Departamento de Zootecnia - UFV, Av. PH Rolfs, Viçosa - MG, CEP: 36571-000.

4 Instituto de Zootecnia, Av. Rui Barbosa s/n, Colina-SP, CEP: 14770-000.

5 Instituto de Zootecnia, Rua Heitor Penteado 56 Centro, Nova Odessa, SP. CEP: 13460-000, CxPO: 60.

${ }^{6}$ Instituto de Zootecnia, CAPTA Bovinos de Corte, Sertãozinho - SP. Bolsista do CNPq.

7 Pós-graduação - LZNA/UENF, Campos dos Goytacazes - RJ, CEP: 28013-602.
}

RESUMO - Avaliou-se o desempenho de 56 bovinos machos não-castrados de três grupos genéticos, com idade média de 18 meses, submetidos à alimentação à vontade ou restrita, em confinamento. Doze animais foram abatidos no início do experimento e os demais (16 Nelore selecionados, 12 Nelore não-selecionados e 16 Caracu selecionados com peso vivo médio inicial de 404, 345 e $434 \mathrm{~kg}$, respectivamente) foram distribuídos em delineamento inteiramente casualizado, em esquema fatorial $2 \times 3$, composto por dois níveis de alimentação (restrito = consumo de $65 \mathrm{~g}$ de $\mathrm{MS} / \mathrm{kgPV}$, 75 por dia e ad libitum $=$ fornecimento do alimento duas vezes ao dia) e três grupos genéticos. $\mathrm{O}$ volumoso utilizado foi a silagem de milho e o concentrado foi constituído de milho moído, farelo de algodão, uréia, monensina e mistura mineral, com relação volumoso:concentrado 50:50. O abate foi realizado quando cada animal atingiu $4 \mathrm{~mm}$ gordura subcutânea à altura da 12 a costela, avaliada por ultra-som. Os grupos genéticos apresentaram ganhos médios diários de peso vivo e pesos de corpo vazio e de carcaça semelhantes, não havendo interação grupo genético $\times$ nível de alimentação. Os ganhos de peso vivo e os pesos de corpo vazio e de carcaça foram maiores nos animais alimentados ad libitum. Somente os consumos de matéria seca em $\mathrm{kg} /$ dia sofreram influência tanto do nível de alimentação quanto do grupo genético. Os animais Caracu apresentaram os maiores consumos, seguidos pelos Nelore selecionados. Os Nelores não-selecionados apresentaram melhor eficiência bionutricional e menor custo de produção da arroba que os demais grupos genéticos.

Palavras-chave: confinamento de bovinos, conversão alimentar, eficiência bionutricional, ganho de peso, ingestão

\section{Production and bioeconomic efficiency of Nellore and Caracu bulls selected to weight gain and fed ad libitum or restricted}

ABSTRACT - Production of 56 feedlot bulls from three different genetic groups averaging 18 months of age and fed ad libitum or restricted was evaluated in this trial. Twelve animals were slaughtered at the beginning of the study and were used as references. The remaining 16 genetically selected Nellore, 12 ordinary Nellore, and 16 genetically selected Caracu with initial average weights of 404,345 and $434 \mathrm{~kg}$, respectively, were assigned to a completely randomized design with a $2 \times 3$ factorial arrangement (two feeding levels and three genetic groups). The dietary levels were either restricted $\left(65 \mathrm{~g} \mathrm{DM}^{\mathrm{B}} / \mathrm{BW}^{0.75}\right)$ or ad libitum intake with animals fed twice a day. Corn silage was used as the forage portion of the diet while the concentrate contained ground corn, cottonseed meal, urea, monensin, and mineral mixture in a forage:concentrate ratio of 50:50. The slaughter criterion was four millimeters of subcutaneous fat measured by ultrasound at the $12^{\text {th }}$ rib area. Bulls from the different genetic groups showed similar average daily weight gain, empty body weight gain, and carcass weight gain. However, these same variables were higher on animals fed ad libitum than on those restricted fed. No significant interaction between genetic group and feeding level was observed in this trial. Dry matter intake was affected not only by feeding level but also by genetic group and it was highest on genetically selected Caracu. Ordinary Nellore showed greater bionutritional efficiency and lower production cost compared to bulls from the other two genetic groups.

Key Words: feedlot, feed:gain ratio, bionutritional efficiency, weight gain, intake

Correspondências devem ser enviadas para: anaton@uenf.br 


\section{Introdução}

A avaliação do desempenho animal envolve análises químico-bromatológicas dos alimentos, determinações do consumo de matéria seca (CMS) e do ganho médio diário de pesos vivo (GMD) e de corpo vazio, estudo da eficiência bioeconômica e, em alguns casos, utilização de diferentes planos nutricionais.

De acordo com Mertens (1994), o desempenho animal tem relação direta com o consumo de matéria seca digestível, de modo que até $90 \%$ de sua variação pode ser ocasionada pelo consumo, e, no máximo, $40 \%$ advêm de flutuações na digestibilidade. OCMS, no entanto, varia conforme o animal, o alimento e as condições de alimentação e de clima (Mertens, 1992).

O estudo da eficiência bionutricional (EBN) deve ser incluído nas avaliações de desempenho, pois é importante determinar a eficiência do ganho de peso pelos animais. A EBN é um índice de discriminação utilizado para revelar em que aspecto um grupo de animais é mais eficiente que o outro (Guidoni, 1994). Considerando que em gado de corte estes aspectos estão relacionados à eficiência de utilização do alimento consumido para conversão em ganho de peso, a análise de EBN utiliza as variáveis ganho médio diário (GMD) e consumo de matéria seca (CMS) de forma conjunta, em uma análise bivariada.

Bovinos de grande porte, oriundos de sistemáticos trabalhos de seleção, podem ser mais eficientes em ambientes sem restrição de alimentos que aqueles de mesma raça, porém não melhorados geneticamente. Entretanto, quando há restrições, principalmente nutricionais, devem ser preferidos os animais de menor porte (Berg \& Butterfield, 1976).

Valadares Filho et al. (2001) afirmaram que fatores como idade, peso, subespécie, raça, classe sexual e nível de ingestão de energia conduzem a diferenças nas exigências nutricionais de bovinos. Contudo, acrescenta-se a estes fatores o potencial genético dos animais, embora trabalhos com zebuínos não tenham especificado o grau e o método de seleção a que a população foi submetida. Segundo Nardon (1998), os resultados de trabalhos de melhoramento realizados durante aproximadamente duas décadas têm comprovado maior desempenho na população de bovinos selecionados, porém com maiores exigências nutricionais.

As pesquisas com bovinos, tanto em confinamento quanto em pastejo, ainda não foram suficientes para estabelecer um recurso eficiente para determinar o momento ideal de abate, ou seja, aquele em que o animal apresenta sua melhor eficiência biológica. Barber et al. (1981) e Oliveira et al. (1998), ao trabalharem com animais da raça Nelore, propuseram o peso fixo de $450 \mathrm{~kg}$ para abate, enquanto Ferreira et al. (1998) e Gesualdi Jr. et al. (2000) sugeriram peso fixo de $500 \mathrm{~kg}$ para abate de mestiços Simental x Nelore e Limousin x Nelore, respectivamente, por ser o peso à idade adulta das matrizes desses rebanhos. Segundo Lanna \& Packer (1998), a adoção do peso das matrizes é, geralmente, mais prática, pois utiliza valor em peso, que é muito importante para o estudo da eficiência dos rebanhos até mesmo em diferentes sistemas de produção.

Uma das alternativas atualmente em estudo é o abate pré-fixado de acordo com a espessura mínima de gordura exigida pelos frigoríficos, em torno de $4 \mathrm{~mm}$. Neste caso, a medida por ultra-som possibilita avaliações periódicas sem a necessidade do abate, porém, mais pesquisas são necessárias para se verificar a eficiência desta técnica e propor os ajustes necessários.

Neste estudo, objetivou-se avaliar o desempenho produtivo e a eficiência bioeconômica de bovinos Nelore e Caracu resultantes de 19 anos de seleção, em condições brasileiras, comparados a bovinos Nelore não-selecionados, todos submetidos à alimentação restrita ou à vontade.

\section{Material e Métodos}

O trabalho foi conduzido na Estação Experimental de Zootecnia de Colina, unidade pertencente ao Instituto de Zootecnia, localizada na região norte do Estado de São Paulo, à altitude média de $589 \mathrm{~m}$, latitude sul de $20^{\circ} 43^{\prime} 5^{\prime \prime} \mathrm{e}$ longitude oeste de $48^{\circ} 32^{\prime} 38^{\prime}$ '.

O clima é classificado, segundo Köppen, como AW, caracterizado como tropical úmido, com estação chuvosa de outubro a março e seca de abril a setembro. A temperatura média do mês mais quente é superior a $22^{\circ} \mathrm{C}$ e a do mês mais frio, superior a $18^{\circ} \mathrm{C}$.

Os 56 tourinhos utilizados foram originados da $19^{\text {a }}$ progênie dos rebanhos Nelore (NeS) e Caracu (CaS) submetidos à seleção com base no peso aos 378 dias de idade (P378) ao final da prova de ganho de peso (PGP), para os machos, e no peso aos 550 dias de idade, para as fêmeas. Utilizou-se, ainda, o rebanho Nelore no qual não foi aplicado o método de seleção $(\mathrm{NeN})$. Todos os rebanhos foram criados na Estação Experimental de Zootecnia de Sertãozinho/IZ.

O período experimental iniciou-se em junho de 2001 e teve a duração marcada pelo tempo de acabamento dos animais, de modo que, quando os animais atingiam no mínimo 4 mm de espessura de gordura subcutânea, avaliada por ultra-som, eram abatidos. O abate do último grupo de animais ocorreu em setembro de 2001. 
Antes do período de adaptação, os animais foram pesados, após jejum completo de 18 horas, identificados com brincos e tratados contra ecto e endoparasitas. Durante o período de adaptação (28 dias), todos os animais receberam a mesma ração utilizada no período experimental.

Após o período de adaptação, 12 animais (quatro de cada grupo genético) foram abatidos, servindo de referência para os estudos subseqüentes. Os 44 animais restantes (16 $\mathrm{NeS}, 12 \mathrm{NeN}$ e $16 \mathrm{CaS}$, com peso vivo médio inicial de 404 , 345 e $434 \mathrm{~kg}$, respectivamente) foram submetidos ao ensaio de alimentação, sendo distribuídos nas baias (individuais), conforme a raça. As baias tinham área total de $30 \mathrm{~m}^{2}\left(8 \mathrm{~m}^{2}\right.$ de área coberta) e eram providas de comedouro e bebedouro de concreto.

Ao início do experimento, efetuaram-se a pesagem inicial dos animais, após jejum completo de 18 horas, e a avaliação da condição corporal via ultra-som e escore corporal, utilizando-se escala de pontuação de 1 a 9. Metade dos animais de cada grupo genético (oito $\mathrm{NeS}$, seis $\mathrm{NeN}$ e oito $\mathrm{CaS}$ ) recebeu alimentação ad libitum até o abate e a outra metade, alimentação restrita, visando fixar o consumo em $65 \mathrm{~g}$ de matéria seca por unidade de tamanho metabólico (65 g de MS/kg/PV ${ }^{0,75}$ ), para se obterem ganhos médios diários moderados e caracterizar baixo plano nutricional.

As pesagens e as medidas de ultra-som e escore corporal foram efetuadas no início do período de adaptação e a cada 28 dias.

A medida ultra-sônica da área de olho-de-lombo e da espessura de gordura subcutânea foi determinada entre a $12^{\mathrm{a}}$ e a 13 a costelas, nos animais contidos em um tronco equipado com tesoura para preensão da cabeça.

A avaliação do peso final no dia do abate foi feita nos animais em jejum completo de 18 horas. Para cada raça, à medida em que um animal do grupo ad libitum era abatido, outro do grupo restrito, da mesma raça, com peso e condição corporal inicial semelhante, também era abatido.

A ração experimental foi balanceada com silagem de milho, milho moído, farelo de algodão, uréia, monensina e mistura mineral e apresentou relação volumoso:concentrado 50:50, avaliada para energia e proteína pelo sistema Cornell Net Carbohydrate and Protein System (CNCPS), versão 4.0 (Barry et al. 1994).

Outros 12 animais, quatro de cada grupo genético (NeS, $\mathrm{NeN} \mathrm{e} \mathrm{CaS}$ ), provenientes da mesma fazenda e com a mesma idade e condição corporal daqueles usados no experimento, foram submetidos, durante sete dias, a um ensaio de digestibilidade. Utilizaram-se gaiolas de metabolismo confeccionadas em madeira, dotadas de comedouro e bebedouro individuais e reguláveis segundo o tamanho do animal.
Durante o período de adaptação (28 dias), os animais foram mantidos em baias individuais iguais às do experimento $\mathrm{e}$ receberam a mesma dieta experimental. No decorrer do ensaio de digestibilidade, dois animais de cada grupo genético foram alimentados à vontade e dois receberam ração restrita.

Coletaram-se amostras de fezes dos animais, do alimento fornecido e das sobras. Iniciou-se a coleta de fezes 48 horas após o início do ensaio, utilizando-se caixotes de madeira adaptados na parte posterior das gaiolas. Posteriormente, as amostras de fezes foram pré-secas, trituradas em moinho com peneira de malha de $1 \mathrm{~mm}$ e agrupadas de forma proporcional, constituindo uma amostra composta, que foi homogeneizada para retirada de uma alíquota representativa, sendo analisada juntamente com as amostras dos alimentos e das sobras.

O teor de NDT determinado no concentrado (79\% na MS) foi obtido utilizando-se a equação NDT $(\mathrm{g} / \mathrm{dia})=$ (proteína dieta - proteína fezes) + (carboidratos dieta carboidratos fezes) $+2,25 \times$ (gordura dieta - gordura fezes) (Sniffen et al., 1992) e dividindo-se o valor encontrado pelo consumo diário médio de matéria seca dos animais (CMS). O teor de energia metabolizável no concentrado foi de $2,85 \mathrm{Mcal} / \mathrm{kg}$ MS. Consta na Tabela 1 a proporção dos ingredientes na mistura do concentrado.

A dieta foi reajustada semanalmente, com base no consumo de MS e na porcentagem de MS do volumoso e do concentrado, e fornecida em duas refeições diárias, às $7 \mathrm{e}$ $16 \mathrm{~h}$. O consumo de alimento foi controlado diariamente, procurando-se manter as sobras em torno de $5 \%$ do fornecido para os animais do grupo ad libitum.

Amostras semanais dos alimentos fornecidos e das sobras foram coletadas para formação de amostras compostas a cada quatro semanas, para determinação dos teores de MS, PB, EE, FDN, FDA, LIG e CIN, conforme metodologias descritas por Silva \& Queiroz (2002) (Tabela 2).

Por ocasião do abate, foram obtidas amostras de couro, pés e cabeça, rúmen-retículo, omaso, abomaso, intestino delgado, intestino grosso vazio, gordura interna, coração, fígado, rins, baço, pulmões, língua, sangue, mesentério e cauda, além de esôfago, traquéia e aparelho reprodutor, os quais foram pesados em conjunto.

O peso corporal vazio (PCVZ) dos animais-referência foi determinado somando-se o peso de carcaça, sangue, cabeça, pés, couro, cauda, vísceras e órgãos. O valor obtido para cada raça dos animais-referência foi utilizado para se estimar o PCVZ inicial dos animais experimentais. O PCVZ final foi determinado por ocasião do abate, de modo semelhante ao obtido para os animais-referência. 
Tabela 1 - Proporção dos ingredientes na mistura do concentrado Table 1 - Ingredient composition of concentrate

\begin{tabular}{|c|c|}
\hline $\begin{array}{l}\text { Ingrediente } \\
\text { Ingredient }\end{array}$ & $\begin{array}{c}\text { Ingrediente na mistura } \\
\text { Ingredient }(\%)\end{array}$ \\
\hline Fubá de milho (\%) & 79,18 \\
\hline \multicolumn{2}{|l|}{ Ground corn (\%) } \\
\hline Farelo de algodão (\%) & 17,18 \\
\hline \multicolumn{2}{|l|}{ Cottonseed meal (\%) } \\
\hline Uréia (\%) & 1,88 \\
\hline \multicolumn{2}{|l|}{ Urea (\%) } \\
\hline Monensina (\%) & 0,054 \\
\hline \multicolumn{2}{|l|}{ Monensin (\%) } \\
\hline Calcário calcítico $(\%)$ & 0,99 \\
\hline \multicolumn{2}{|l|}{ Limestone (\%) } \\
\hline Sal comum $(\%)$ & 0,70 \\
\hline \multicolumn{2}{|l|}{ Salt $(\%)$} \\
\hline Sulfato de zinco $(\mathrm{g} / 100 \mathrm{~kg})$ & 13,24 \\
\hline \multicolumn{2}{|l|}{ Zinc sulfate $(\mathrm{g} / 100 \mathrm{~kg})$} \\
\hline Selenito de sódio $(\mathrm{g} / 100 \mathrm{~kg})$ & 0,034 \\
\hline \multicolumn{2}{|l|}{ Sodiun selenite $(\mathrm{g} / 100 \mathrm{~kg})$} \\
\hline Sulfato de manganês $(\mathrm{g} / 100 \mathrm{~kg})$ & 6,24 \\
\hline Manganese sulfate $(\mathrm{g} / 100 \mathrm{~kg})$ & \\
\hline
\end{tabular}

O peso inicial de carcaça dos animais experimentais também foi estimado utilizando-se os valores obtidos dos animais-referência.

O ganho médio diário de corpo vazio (GMCVZ) foi obtido pela divisão da diferença entre os valores finais e iniciais do PCVZ pelo número de dias em que cada animal permaneceu no experimento. O ganho de carcaça (GCAR) foi obtido da mesma maneira, utilizando-se os pesos finais e iniciais de carcaça.

Para obtenção dos valores de eficiência bionutricional $(\mathrm{EBN})$, as variáveis ganho médio diário (GMD) e consumo de matéria seca(CMS) foram consideradas de forma conjunta em análise de variância bidimensional (Pimentel Gomes, 2000), segundo delineamento inteiramente casualizado, desconsiderando-se o arranjo fatorial.

A partir das informações geradas pela MANOVA, obtiveram-se os autovalores associados à matriz de soma de quadrados de "tratamentos" por meio da equação característica (Harris, 1975):

$$
\operatorname{det}\left[E^{-1} H-\lambda I\right]=0
$$

em que: $E^{-1}=$ inversa comum da matriz de somas de quadrados residuais; $\mathrm{H}=$ matriz de somas de quadrados para "tratamentos"; $\lambda=$ autovalor canônico; e I = matriz identidade de ordem $n=2$.

A partir do maior autovalor canônico obtido, estimou-se o autovetor não-normalizado correspondente por meio da solução do sistema de equações (Detmann et al., 2005):

$$
\left(E^{-1} H-\lambda_{1} I\right) \tilde{v}=\left[\begin{array}{l}
0 \\
0
\end{array}\right] \Rightarrow\left(E^{-1} H\right) \tilde{v}=\lambda_{1} \tilde{v} \Rightarrow\left(E^{-1} H\right)\left[\begin{array}{l}
a \\
b
\end{array}\right]=\lambda_{1}\left[\begin{array}{l}
a \\
b
\end{array}\right]
$$

em que: $\lambda_{1}=$ maior autovalor canônico; $\widetilde{v}=$ autovetor nãonormalizado associado ao maior autovalor canônico; a e b = coeficientes canônicos; e $\mathrm{E}^{-1}, \mathrm{H}$ e I = como definidos anteriormente.

Posteriormente, procedeu-se à normalização do autovetor obtido pela solução do sistema linear conforme a restrição:

$$
\widetilde{v}_{n}^{\prime} E \widetilde{v}_{n}=1 \Rightarrow\left[\begin{array}{ll}
a^{\prime} & b^{\prime}
\end{array}\right] E\left[\begin{array}{l}
a^{\prime} \\
b^{\prime}
\end{array}\right]=1
$$

em que: $\widetilde{v}_{n}=$ autovetor normalizado associado ao maior autovetor canônico; a' e b' = coeficientes canônicos ajustados pela normalização do vetor $\widetilde{v} ; \mathrm{e} \mathrm{E}=$ matriz de soma de quadrados residuais.

A partir dos coeficientes do autovetor canônico normalizado definido na equação anterior, produziu-se a primeira variável discriminante canônica ou eficiência bionutricional (EBN), definida como:

$E B N=a^{\prime} \cdot C M S+b^{\prime} \cdot G M D=0,2073 \cdot C M S-0,0565 \cdot G M D$ em que: $\mathrm{CMS}=$ consumo voluntário de matéria seca $(\mathrm{kg} / \mathrm{dia})$; $\mathrm{GMD}=$ ganho médio diário $(\mathrm{kg} / \mathrm{dia})$.

\begin{tabular}{|c|c|c|c|c|c|}
\hline $\begin{array}{l}\text { Nutriente } \\
\text { Nutrient }\end{array}$ & $\begin{array}{l}\text { Mistura concentrada } \\
\text { Concentrate mixture }\end{array}$ & $\begin{array}{c}\text { Farelo de algodão } \\
\text { Cottonseed meal }\end{array}$ & $\begin{array}{c}\text { Fubá de milho } \\
\text { Ground corn }\end{array}$ & $\begin{array}{c}\text { Silagem de milho } \\
\text { Corn silage }\end{array}$ & $\begin{array}{c}\text { Dieta } \\
\text { Diet }\end{array}$ \\
\hline $\operatorname{MS}(\%)(D M, \%)$ & 81,70 & 81,94 & 79,57 & 27,14 & 54,42 \\
\hline $\mathrm{MO}^{1}(O M)$ & 95,29 & 94,50 & 98,55 & 95,95 & 95,61 \\
\hline $\mathrm{PB}^{1}(C P)$ & 18,81 & 34,40 & 9,46 & 8,82 & 13,81 \\
\hline $\mathrm{FDN}^{1}(N D F)$ & 15,98 & 36,96 & 12,56 & 60,92 & 38,45 \\
\hline FDNcp $^{1}$ (NDFap) & 13,97 & 33,98 & 11,06 & 57,69 & 35,82 \\
\hline $\mathrm{CHOT}^{2}(T C)$ & 72,67 & 55,92 & 86,06 & 84,88 & 78,77 \\
\hline $\mathrm{EE}^{1}(E E)$ & 3,81 & 4,18 & 3,03 & 2,25 & 3,02 \\
\hline $\mathrm{NDT}^{4}(T D N)$ & - & - & - & - & 79,00 \\
\hline
\end{tabular}

Tabela 2 - Composição química do concentrado, da silagem de milho e da dieta e NDT da dieta Table 2 - Chemical composition of concentrate, corn silage and diet and total digestible nutrient (TDN) of diet

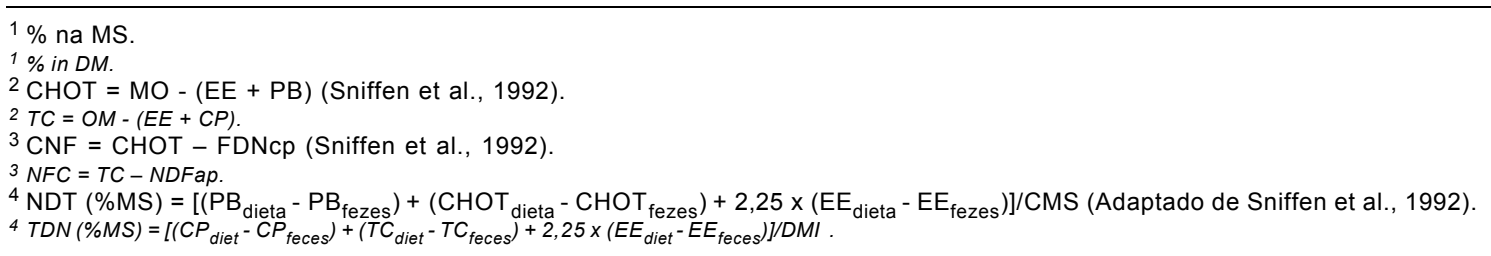


A EBN, em conjunto com as demais variáveis, foi submetida à análise de variância univariada, segundo delineamento inteiramente casualizado em arranjo fatorial 2 × 3 composto por dois níveis de alimentação (restrito e outro $a d$ libitum) e três grupos genéticos (Nelore selecionado, Nelore não-selecionado e Caracu selecionado). Para comparação das médias, utilizou-se o teste de Diferença Mínima Significativa, adotando-se $\alpha=0,05$.

\section{Resultados e Discussão}

Não houve interação de grupo genético e nível de alimentação para nenhum dos fatores estudados $(\mathrm{P}>0,05)$. As médias de mínimos quadrados para ganho médio diário (GMD) e ganhos de peso de corpo vazio (GMCVZ) e de peso de carcaça (GCAR), consumos de matéria seca (CMS) e fibra em detergente neutro (CFDN) são apresentadas na Tabela 3. Para os grupos selecionados, foram discutidas as diferenças entre $\mathrm{NeS}$ e $\mathrm{CaS}$ e as comparações dos grupos selecionados e não-selecionados foram feitas entre os animais $\mathrm{NeS}$ e $\mathrm{NeN}$.

Somente o nível de alimentação influenciou os GMD, GMCVZ e GCAR. A ausência de diferença estatística entre os grupos genéticos para GMD, GMCVZ, GCAR e CMS $(\% \mathrm{PV})$ pode estar relacionada ao fato de os animais estarem em terminação, ou seja, em estágio avançado de desenvolvimento, e terem sido abatidos preconizando-se o mesmo grau de acabamento. Estes resultados corroboram parcialmente os de Nardon (1998), que encontrou CMS, em \%PV, semelhante para $\mathrm{NeS}$ e $\mathrm{NeN}$ e para $\mathrm{NeS}$ e $\mathrm{CaS}$. Ainda segundo este autor, os GMD foram semelhantes entre grupos genéticos selecionados, mas foram superiores para os $\mathrm{NeS}$ em relação aos $\mathrm{NeN}$.
Os GMD registrados neste estudo, em torno de $1,00 \mathrm{~kg} / \mathrm{dia}$ para os animais com alimentação ad libitum, podem ser considerados baixos para bovinos de corte em confinamento e podem ter sido determinados pelo baixo CMS como \% do $\mathrm{PV}$, que ficou abaixo de $2 \%$.

O CMS, em kg/dia, foi influenciado $(\mathrm{P}<0,05)$ pelo grupo genético. Os animais $\mathrm{CaS}$ apresentaram os maiores consumos, seguidos pelos NeS. Animais com maior potencial para crescimento muscular podem apresentar maior consumo de alimentos. Segundo Owens et al. (1993), as diferenças na composição corporal ocasionam ingestão diretamente proporcional à massa protéica e inversamente proporcional à deposição de gordura. Assim, bovinos que entram no confinamento com peso maior, em razão do maior desenvolvimento da massa muscular, geralmente apresentam maior consumo de alimentos, especialmente quando são de grande porte ou posuem peso corporal maduro. Entretanto, o con sumo de alimentos diminui quando o teor de gordura aumenta, assim como ocorre com o avanço do período de terminação.

O maior CMS encontrado para $\mathrm{NeS}$ em relação aos $\mathrm{NeN}$ pode ser explicado pela maior massa corporal dos $\mathrm{NeS}$, evidenciada pelas diferenças no PVI (404 kg para NeS e $345 \mathrm{~kg}$ para $\mathrm{NeN}$ ), e, posteriormente, pelas diferenças no PVA (493 kg para NeS e $424 \mathrm{~kg}$ para NeN)(Tabela 4), sugerindo que a demanda de produção aumentada, no caso dos animais selecionados, pode ter afetado o consumo de alimentos.

O CFDN (\% do PV) não foi influenciado pelo nível de alimentação ou pelos grupos genéticos, o que era esperado, visto que, em termos qualitativos, a dieta foi a mesma para todos os animais. O teor de FDN da dieta, de 38,45\% na MS (Tabela 2), não ocasionou problemas no consumo de alimentos, como regulação metabólica, mesmo em animais zebuínos, considerados mais sensíveis a baixos níveis de FDN.

Tabela 3 - Médias de mínimos quadrados para ganhos diários de peso vivo (GMD), de peso corporal vazio (GMCVZ) e de peso de carcaça (GCAR) e consumos de MS (CMS) e FDN (CFDN), para os grupos genéticos e nível de alimentação

Table 3 - Least square means of average daily gain (ADG), empty body gain (EBG) and carcass gain (CARG), dry matter intake (DMI) and neutral detergent fiber intake (NDFI) of bulls from different genetic groups fed ad libitum or restricted

\begin{tabular}{|c|c|c|c|c|c|c|}
\hline \multirow[t]{2}{*}{$\begin{array}{l}\text { Item } \\
\text { Item }\end{array}$} & \multicolumn{2}{|c|}{$\begin{array}{c}\text { Nível de alimentação } \\
\text { Nutritional level }\end{array}$} & \multicolumn{3}{|c|}{$\begin{array}{l}\text { Grupo genético }{ }^{1} \\
\text { Genetic group }\end{array}$} & \multirow[t]{2}{*}{ CV $(\%)$} \\
\hline & $\begin{array}{l}\text { Ad libitum } \\
\text { Ad libitum }\end{array}$ & $\begin{array}{l}\text { Restrito } \\
\text { Restrict }\end{array}$ & $\begin{array}{l}\mathrm{NeS} \\
G S N\end{array}$ & $\begin{array}{c}\mathrm{NeN} \\
O N\end{array}$ & $\begin{array}{l}\mathrm{CaS} \\
G S C\end{array}$ & \\
\hline $\operatorname{GMD}(\mathrm{kg} / \mathrm{dia})(A D G, \mathrm{~kg} /$ day $)$ & $1,005 \mathrm{a}$ & $0,675 b$ & $0,885 \mathrm{a}$ & $0,841 \mathrm{a}$ & $0,793 \mathrm{a}$ & 23,81 \\
\hline $\operatorname{GMCVZ}(\mathrm{kg} / \mathrm{dia})(E B G, \mathrm{~kg} /$ day $)$ & $1,221 \mathrm{a}$ & $0,690 \mathrm{~b}$ & $0,980 \mathrm{a}$ & $0,956 \mathrm{a}$ & $0,930 \mathrm{a}$ & 30,04 \\
\hline $\operatorname{GCAR}(\mathrm{kg} / \mathrm{dia})(C A R G, \mathrm{~kg} /$ day $)$ & $0,812 \mathrm{a}$ & $0,516 b$ & $0,682 \mathrm{a}$ & $0,715 \mathrm{a}$ & $0,596 \mathrm{a}$ & 31,77 \\
\hline CMS (\%PV) (DMI, \%BW) & $1,71 \mathrm{a}$ & $1,06 \mathrm{~b}$ & $1,37 \mathrm{a}$ & $1,41 \mathrm{a}$ & $1,39 \mathrm{a}$ & 8,70 \\
\hline CMS $\left(\mathrm{g} / \mathrm{kg}^{0,75}\right)\left(D M I, g / \mathrm{kg}^{0.75}\right)$ & $81,22 \mathrm{a}$ & $65,00 \mathrm{~b}$ & $64,62 \mathrm{a}$ & $64,09 a$ & $66,60 \mathrm{a}$ & 8,67 \\
\hline CFDN (\%PV) (DMI, \%BW) & $0,74 \mathrm{a}$ & $0,71 \mathrm{a}$ & $0,78 \mathrm{a}$ & $0,73 \mathrm{a}$ & $0,67 \mathrm{a}$ & 17,61 \\
\hline
\end{tabular}

${ }_{1}^{1} \mathrm{NeS}$ : Nelore selecionado(GSN: genetically selected Nellore); NeN: Nelore não-selecionado(ON: ordinary Nellore); CaS: Caracu selecionado (GSC: genetically selected Caracu).

Médias seguidas pela mesma letra em cada linha, em cada parâmetro, não diferem $(P>0,05)$ pelo teste DMS

Means followed by the same letter in the same row do not differ $(P>0.05)$ by LSD test. 
É necessário, no entanto, avaliar se bovinos Nelore selecionados podem apresentar maior desempenho e eficiência que os não-selecionados, caso seja empregado nível superior a $50 \%$ de concentrado na dieta deste grupo genético.

Uma vez que essas variáveis são influenciadas pelas espécies de forrageiras e considerando-se que, neste caso, foram utilizadas plantas tropicais, com grande capacidade de limitar o consumo, em decorrência de fatores físicos, resultados divergentes podem ainda ser encontrados na literatura brasileira. Além disso, as forrageiras de clima tropical encontram-se em ambiente de maior biodiversidade, onde existe maior interação de seres vivos e de seres e ecossistema, o que determina diferenças na qualidade dos alimentos produzidos em uma fazenda em relação a outra, dificultando a obtenção de resultados coerentes entre os trabalhos.

As médias de mínimos quadrados para peso vivo ao abate (PVA), conversão alimentar (CA) e eficiência bionutricional (EBN) e as médias aritméticas para dias de confinamento (DC) são apresentadas na Tabela 4.

A CA sofreu influência somente do grupo genético, de modo que os animais Caracu apresentaram os maiores valores $(10,19)$, superiores ao observado por Nardon (1998) neste mesmo grupo genético. A conversão alimentar dos animais $\mathrm{NeS}(7,75)$ pode ser considerada melhor que a de 8,36 relatada por Nardon (1998) para NeS resultantes de quatro progênies anteriores a esta.

A tendência de EBN foi oposta ao desempenho produtivo animal, ou seja, a melhor eficiência de produção coincidiu com a menor estimativa de EBN. Segundo Detmann et al. (2005), esse comportamento é normalmente observado em razão da natureza exclusivamente discriminatória da EBN, não desqualificando, contudo, as inferências obtidas.

Os animais com alimentação restrita apresentaram melhor EBN que aqueles do tratamento ad libitum. Os requerimentos de energia metabolizável para mantença $\left(\mathrm{REM}_{\mathrm{m}}\right)$ e os de eficiência de uso da energia metabolizável para mantença $\left(\mathrm{k}_{\mathrm{m}}\right)$ determinam a eficiência nutricional dos animais e aqueles que recebem alimentação restrita tendem a apresentar melhores valores para este parâmetro que os alimentados à vontade.

Ledger \& Sayers (1977) demonstraram que a exigência de mantença é reduzida após períodos de redução do consumo e as bases metabólicas para estas mudanças incluem alterações nas taxas de bombeamento de íons e na ciclagem de metabólitos, além de alterações no tamanho e nas taxas metabólicas de órgãos viscerais. Assim, as pesquisas comprovam grande variação na extensão desta redução, que pode ser de 10 até $50 \%$, e provavelmente os valores para EBN de animais recebendo alimentação restrita dependerá desse intervalo de grandezas.

Quanto à EBN, neste estudo, os NeN foram mais eficientes que os $\mathrm{NeS}$, que, por sua vez, foram mais eficientes que os $\mathrm{CaS}(\mathrm{P}<0,05)$. É provável que o nível de concentrado $(50 \%)$ na dieta tenha sido insuficiente para promover GMD superiores para os $\mathrm{NeS}$, o que promoveu $\mathrm{EBN}$ inferior à dos $\mathrm{NeN}$, que apresentaram menor CMS e mesmo GMD $(\mathrm{P}<0,05)$.

Owens et al. (1993) afirmaram que os bovinos submetidos ao confinamento com maior peso, como é o caso dos animais selecionados deste estudo, normalmente apresentam maior ingestão de alimentos, especialmente quando são de conformação grande e de maior peso adulto, situação verificada quando foram comparados os pesos vivos iniciais (PVI) dos animais selecionados ou dos não-selecionados. Ressalta-se que essa situação se refere a uma condição experimental, mas permite inferir que a campo seria recomendável confinar os animais com pesos inferiores a $400 \mathrm{~kg}$, mesmo aqueles selecionados para grande porte.

Semelhantemente aos GMD, os GMCVZ também foram iguais $(\mathrm{P}>0,05)$ para os animais deste estudo. A melhor forma de avaliar as diferenças no desempenho animal é por meio do GMCVZ, pois segundo Owens et al. (1995), este

Tabela 4 - Médias de mínimos quadrados para peso vivo ao abate (PVA), conversão alimentar (CA) e eficiência bionutricional (EBN) e médias aritméticas com erro-padrão dos dias de confinamento (DC) para os grupos genéticos e os níveis de alimentação

Table 4 - Least square means of slaughter weight (SW), feed:gain ratio ( $F / G)$, bionutritional efficiency (BE) and average confinement days (CD) of bulls from different genetic groups fed ad libitum or restricted

\begin{tabular}{|c|c|c|c|c|c|c|}
\hline \multirow[t]{2}{*}{$\begin{array}{l}\text { Item } \\
\text { Item }\end{array}$} & \multicolumn{2}{|c|}{$\begin{array}{c}\text { Nível de alimentação } \\
\text { Nutritional level }\end{array}$} & \multicolumn{3}{|c|}{$\begin{array}{l}\text { Grupo genético }{ }^{1} \\
\text { Genetic group }\end{array}$} & \multirow[t]{2}{*}{ CV (\%) } \\
\hline & $\begin{array}{l}\text { Ad libitum } \\
\text { Ad libitum }\end{array}$ & $\begin{array}{c}\text { Restrito } \\
\text { Restrict }\end{array}$ & $\begin{array}{l}\mathrm{NeS} \\
G S N\end{array}$ & $\begin{array}{c}\mathrm{NeN} \\
O N\end{array}$ & $\begin{array}{l}\mathrm{CaS} \\
G S C\end{array}$ & \\
\hline PVA $(\mathrm{kg})(S W, k g)$ & $512 \mathrm{a}$ & $450 \mathrm{~b}$ & $493 b$ & $424 \mathrm{c}$ & $526 \mathrm{a}$ & 7,80 \\
\hline $\mathrm{CA}(F / G)$ & $9,09 \mathrm{a}$ & $7,70 \mathrm{a}$ & $7,75 b$ & $7,23 b$ & $10,19 a$ & 32,19 \\
\hline $\mathrm{EBN}(B E)$ & $1,86 \mathrm{a}$ & $1,02 b$ & $1,46 \mathrm{~b}$ & $1,30 \mathrm{c}$ & $1,58 \mathrm{a}$ & 11,11 \\
\hline $\mathrm{DC}(C D)$ & $116 \pm 5,72$ & $116 \pm 5,72$ & $114 \pm 10,14$ & $104 \pm 10,47$ & $130 \pm 6,92$ & - \\
\hline
\end{tabular}

${ }^{1} \mathrm{NeS}$ : Nelore selecionado(GSN: genetically selected Nellore); NeN: Nelore não-selecionado(ON: ordinary Nellore); CaS: Caracu selecionado (GCS: genetically selected Caracu).

Médias seguidas pela mesma letra em cada linha não diferem $(P>0,05)$ pelo teste $D M S$

Means followed by the same letter in the same row do not differ $(P>0.05)$ by LSD test. 
parâmetro anula os efeitos do peso do conteúdo do trato gastrintestinal.

O GCAR reflete a eficiência do sistema na obtenção do produto que será comercializado. Os valores encontrados $(0,68 ; 0,71$ e $0,59 \mathrm{~kg} /$ dia para $\mathrm{NeS}, \mathrm{NeN}$ e CaS, respectivamente) foram semelhantes entre si, porém inferiores ao relatados por Jorge et al. (1998), de 1,12 kg, e Oliveira et al. (1998), de 0,84 kg, para animais Nelore, mas foram superiores aos reportados por Nardon (1998), de 0,50; 0,44 e 0,49 kg, respectivamente, para $\mathrm{NeS}, \mathrm{NeN}$ e $\mathrm{CaS}$.

Os dados divergentes da literatura, para bovinos de mesmo grupo genético, podem estar relacionados aos diferentes pesos vivos iniciais e finais dos animais empregados nos experimentos, visto que, segundo Fitzhugh (1976), o desempenho animal é influenciado por esses dois fatores.

Os pesos vivos de abate (PVA) foram maiores para os animais $\mathrm{CaS}$ que para os $\mathrm{NeS}$, observando-se diferença significativa entre os Nelore, de modo que os $\mathrm{NeS}$ foram, em média, $69 \mathrm{~kg}$ mais pesados ao abate. Considerando que ao abate, os animais $\mathrm{NeS}$ e NeN apresentavam mesmo grau de acabamento,verificou-se que a seleção para peso aos 378 dias de idade proporcionou ganho genético na raça Nelore, obtendo-se animais mais pesados ao abate.

Constam na Tabela 5 os custos de produção, nos quais utilizaram-se as médias do custo por animal por dia de confinamento multiplicadas pelas médias de dias de confinamento para cada grupo genético. $\mathrm{O}$ valor obtido foi dividido pelo ganho médio total de carcaça, em arrobas, durante o confinamento, utilizando-se para este cálculo apenas os animais alimentados à vontade.

$O$ custo de produção da arroba superior ao valor de mercado para os animais $\mathrm{CaS}$ decorreu do alto custo diário da alimentação, ocasionado pelo elevado CMS e pelo extenso período de confinamento. Deve-se considerar ainda que esta é uma raça tardia e, conforme mencionado anteriormente, houve expectativa de atingir $4 \mathrm{~mm}$ de espessura de gordura subcutânea, medida pela técnica de ultra-som, para se realizar o abate. Em situações de campo, pode-se recomendar a castração ou o fornecimento de rações com maiores teores de concentrado ou, ainda, a combinação dos dois fatores para reduzir o período necessário para abate de animais tardios.

Tabela 5 - Custos de produção da arroba produzida durante o confinamento de bovinos machos não-castrados de diferentes grupos genéticos recebendo alimentação à vontade

Table 5 - Production costs of arroba during confinement of bulls from different genetic groups fed ad libitum or restricted

\begin{tabular}{|c|c|c|c|c|c|}
\hline $\begin{array}{l}\text { Grupo genético }{ }^{1} \\
\text { Genetic group }\end{array}$ & $\begin{array}{l}\text { Custo/animal/dia } \\
\text { (U\$) } \\
\text { Cost/animal/day (U\$) }\end{array}$ & $\begin{array}{c}\text { Dias de confinamento } \\
\text { Confinement days }\end{array}$ & $\begin{array}{c}\text { Ganho total de } \\
\text { carcaça, @ } \\
\text { Total carcass gain, @ }\end{array}$ & $\begin{array}{c}\text { Custo/@produzida } \\
(\mathrm{U} \$)^{2} \\
\text { Cost } @ @ \text { produced }(U \$)^{2}\end{array}$ & $\begin{array}{c}\text { Custo/@produzida } \\
\text { (R\$) } \\
\text { Cost } @ \text { produced (R\$) }\end{array}$ \\
\hline $\mathrm{NeS}(G S N)$ & 0,80 & 114 & 6,7 & 13,61 & 43,55 \\
\hline $\mathrm{NeN}(O N)$ & 0,71 & 104 & 5,8 & 12,73 & 40,73 \\
\hline $\mathrm{CaS}(G S C)$ & 0,87 & 130 & 6,3 & 17,95 & 57,44 \\
\hline
\end{tabular}

${ }^{1} \mathrm{NeS}$ : Nelore selecionado(GSN: genetically selected Nellore); NeN: Nelore não-selecionado(ON: ordinary Nellore); CaS: Caracu selecionado (GCS: genetically selected Caracu).

2 Valores calculados multiplicando-se a média do custo/animal/dia pela média dos dias de confinamento e dividindo-se pela média do ganho total de carcaça em @, durante o confinamento. O valor de mercado considerado para a @ foi de R\$54,00

2 Calculated by multiplying the average cost/animal/day by the average of confinement days and dividing by the total carcass gain in @, during the confinement. The market value used for @ was of $R \$ 54.00$.

As diferenças na composição química, calculada pelos ganhos em função do peso e da idade dos animais durante o confinamento, refletem sobre os custos de produção. Segundo Lanna \& Packer (1998), a deposição de proteína é menos eficiente energeticamente (Mcal/Mcal) que a de gordura, porém é mais eficiente em peso de tecido depositado (kg músculo/Mcal ingerida), uma vez que, para cada unidade de ganho de proteína, aproximadamente três unidades de água são depositadas. Portanto, o custo do ganho de peso aumenta à medida que o animal se torna mais gordo ou que o período de terminação é estendido. De acordo com esses autores, animais de maior tamanho corporal são mais eficientes apenas quando abatidos ao mesmo peso de animais de menor tamanho, pois depositam menos gordura no ganho. Entretanto, quando o abate é realizado para produção de carcaças com o mesmo teor de gordura, animais de menor tamanho corporal são mais eficientes, pois diluem as exigências de mantença.

\section{Conclusões}

Os animais Nelore não-selecionados foram mais eficientes em termos bionutricionais, pois o consumo foi menor e o ganho de peso foi o mesmo que o dos Nelore selecionados.

Sem avaliação da eficiência bionutricional, os animais recebendo alimentação à vontade foram mais eficientes que aqueles sob alimentação restrita, visto que os ganhos de peso vivo de peso corporal vazio e de carcaça foram maiores.

Os bovinos Caracu selecionados foram superiores apenas no ganho de carcaça e no peso vivo ao abate, porém 
apresentaram menor eficiência bionutricional que os zebuínos.

\section{Literatura Citada}

BARBER, K.A.; WILSON, L.L.; ZIEGLER, J.H. et al. Charolais and angus slaughtered at equal percentages of mature cow weight. 1. Effects of slaughter weight and diet energy densit on carcass traits. Journal of Animal Science, v.52, n.2, p.218-231, 1981.

BARRY, M.C.; FOX, D.C.; TYLUTKY, T.P. et al. A manual for using the Cornell Net Carbohydrate and Protein System for evaluating cattle diets. Revised for CNCPS. Release 3.ed. Ithaca: 1994. 40p.

BERG, R.T.; BUTTERFIELD, R.M. New concepts of cattle growth. New York: Sydney University, 1976. 240p.

DETMANN, E.; CECON, P.R.; ANDREOTTI, M.O. et al. Aplicação da primeira variável canônica na avaliação de experimentos de desempenho animal. Revista Brasileira de Zootecnia, v.34, n.6, p.2417-2426, 2005.

FERREIRA, M.A; VALADARES FILHO, S.C.; COELHO DA SILVA, J.F. et al. Consumo, conversão alimentar, ganho de peso e características da carcaça de bovinos $\mathrm{F}_{1}$ Simental x Nelore. Revista Brasileira de Zootecnia, v.28, n.2, p.343-351, 1998.

FITZHUGH, H.A. Analysis of growth curves and strategies for altering their shape. Journal of Animal Science, v.42, n.4, p.1036-1051, 1976

GESUALDI JR., A.; PAULINO, M.F.; VALADARES FILHO, S.C. et al. Níveis de concentrado na dieta de novilhos F1 limousin $\mathrm{x}$ nelore: consumo, conversão alimentar e ganho de peso. Revista Brasileira de Zootecnia, v.29, n.5, p.1458-1466, 2000.

GUIDONI, A.L. Alternativas para comparar tratamentos envolvendo o desempenho nutricional animal. Piracicaba: Escola Superior de Agricultura "Luiz de Queiroz", 1994. 105p. Tese (Doutorado em Agronomia) - Escola Superior de Agricultura "Luiz de Queiroz", 1994.

HARRIS, R.J. A primer of multivariate statistics. New York: Academic Press, 1975. 332p.

JORGE, A.M.; FONTES, C.A.A.; PAULINO, M.F. et al. Desempenho produtivo de animais de quatro raças zebuínas, abatidos em três estágios de maturidade. 1. Ganho de peso e de carcaça e eficiência de ganho. Revista Brasileira de Zootecnia, v.27, n.4, p.766769, 1998.

LANNA, D.P.; PACKER, I.V. Eficiência biológica e econômica de bovinos de corte. In: WORKSHOP SOBRE QUALIDADE DA CARNE E MELHORAMENTO GENÉTICO DE BOVINOS, 1., 1998, São Carlos. Anais... São Carlos: EMBRAPA/FUNDEPEC. 1998. p.83-104.
LEDGER, H.P.; SAYERS, A.R. The utilization of dietary energy by steers during periods of restricted feed intake of subsequent realimentation. 1. The effect of time on the mantenance requirements of steers held at constant live weight. Journal Agricultural Science, v.88, n.1, p.11-26, 1977.

MERTENS, D.R. Análise da fibra e sua utilização na avaliação e formulação de rações. In: REUNIÃO ANUAL DA SOCIEDADE BRASILEIRA DE ZOOTECNIA, 29., 1992, Lavras. Anais... Lavras: Sociedade Brasileira de Zootecnia, 1992. p.188-219.

MERTENS, D.R. Regulation of forage intake. In: FAHEY, G.C., COLLINS, M.; MERTENS, D.R. et al. (Eds). Forage quality evaluation and utilization. Madison: ASA, CSSA, SSSA. p.450-493, 1994.

NARDON, R.F. Seleção de bovinos para desempenho: composição corporal e características de carcaça. Jaboticabal: Universidade Estadual Paulista, 1998. 107p. Tese (Doutorado em Zootecnia) - Universidade Estadual Paulista, 1998.

OLIVEIRA, S.R.; COELHO DA SILVA, J.F.; VALADARES FILHO. S.C. et al. Desempenho de novilhos nelore, não castrados, recebendo rações com vários níveis de concentrado. In: REUNIÃO ANUAL DA SOCIEDADE BRASILEIRA DE ZOOTECNIA, 35., 1998, Botucatu. Anais... Botucatu: Sociedade Brasileira de Zootecnia, 1998. p.155-157.

OWENS, F.N.; DUBESKI, P.; HANSON, C.F. Factors that alter the growth and development of ruminants. Jounal of Animal Science, v.71, n.6, p.3138-3150, 1993.

OWENS, F.N.; GILL, D.R.; SECRIST, D.S. et al. Review of some aspects of growth and development of feedlot catle. Jounal of Animal Science, v.73, n.10 p.3152-3172, 1995.

PIMENTEL GOMES, F. Curso de estatística experimental. 14.ed. Piracicaba: Degaspari, 2000. 477p.

SILVA, D.J.; QUEIROZ, A.C. Análise de alimentos (métodos químicos e biológicos). 3.ed. Viçosa, MG: Universidade Federal de Viçosa, 2002. 235p.

SNIFFEN, C.J.; O'CONNOR, J.D.; Van SOEST, P.J. et al. A net carbohydrate and protein system for evaluating cattle diets: II. Carbohydrate and protein availability. Jounal of Animal Science, v.70, n.11, p.3562-3577, 1992.

VALADARES FILHO, S.C.; SILVA, F.F.; ROCHA JR., V.R. et al. Tabelas de composição de alimentos e exigências nutricionais para bovinos no Brasil. In: II SIMPÓSIO DE PRODUÇÃO DE GADO DE CORTE, 2001, Viçosa, MG. Anais... Viçosa, MG: Universidade Federal de Viçosa, 2001. p.291-358. 